\title{
Composite renal cell carcinoma and angiomyolipoma in a patient with Tuberous sclerosis: A diagnostic dilemma
}

\author{
Aanchal Kakkara, MD; Archana George Vallonthaiel', MD; Mehar Chand Sharma ${ }^{a}$, MD, FRCPath; \\ Girdhar Bora ${ }^{b}$, MCh; Ananya Panda ${ }^{c}, M D ;{ }^{*}$ Amlesh Seth ${ }^{b}, M C h$
}

Departments of aPathology, UUrology and Radiodiagnosis, All India Institute of Medical Sciences, New Delhi, India

Cite as: Can Urol Assoc J 2015;9(7-8):E507-10. http://dx.doi.org/10.5489/cuaj.2532 Published online July 17, 2015.

\section{Abstract}

Tuberous sclerosis (TS) is an autosomal dominant disorder associated with various renal pathologies, including angiomyolipoma (AML). Renal cell carcinoma (RCC) with concomitant AML is rare. We report a case of composite RCC and AML in a young male with TS. This 25-year-old male presented with an abdominal mass. The abdominal computed tomography scan revealed RCC in the left kidney and $\mathrm{AML}$ in right kidney. A left radical nephrectomy was performed. Microscopy showed a tumour composed of closely intermingled RCC and AML components. Immunohistochemistry was performed for confirmation. A diagnosis of composite tumour with clear cell RCC and AML was made. Though the coexistence of RCC with AML is rare, and the composite variant is even rarer, one must be aware of this entity to prevent misdiagnosis as well as upstaging of RCC, and also to avoid missing areas of RCC within a large AML, especially in TS patients.

\section{Introduction}

Angiomyolipoma (AML) with concomitant renal cell carcinoma (RCC) is rare; this rare disease is seen commonly in patients with tuberous sclerosis (TS). To date, there are about 60 reported cases of concomitant AML with RCC, many related to TS. ${ }^{1,2}$

\section{Case Report}

A 25-year-old male, with TS, presented with complaints of progressively increasing abdominal swelling for 2 years. There was history of decreased appetite and significant weight loss during the period of illness. On examination, facial lesions typical of adenoma sebaceum were present. A large, bimanually palpable mass was felt in the left lumbar region. Contrast-enhanced computed tomography $(\mathrm{CT})$ of the abdomen (Fig. 1) revealed bilateral renal masses, suggestive of RCC of the left kidney, and AML in the right kidney. There was no evidence of lymph node metastases. A positron emission tomography scan revealed active disease in the left kidney, with metastatic deposits in bilateral lungs, liver, and right femoral head. Multiple subependymal tubers were identified on brain magnetic resonance imaging (MRI). Ophthalmological examination revealed retinal angiomas. We confirmed a clinical diagnosis of RCC with metastases in this patient with TS. The patient underwent a radical nephrectomy. Grossly, the kidney was markedly enlarged (Fig. 2, part A). No capsular breach was noted. Cut surface showed a large grey-white tumour (Fig. 2, part B) measuring $25 \mathrm{~cm}$, replacing almost the entire kidney, with areas of hemorrhage and necrosis.

Microscopic examination (Fig. 3) showed a tumour composed of two distinct components which were closely intermingled with each other. The first comprised of large polygonal cells with abundant granular eosinophilic cytoplasm, round to oval nuclei with irregular nuclear membranes, coarse chromatin and prominent nucleoli (Fuhrman grade 4), simulating clear cell RCC. The second component showed groups and bundles of spindled to epithelioid cells interspersed among the former. At places, the spindled cells appeared to come from thick-walled blood vessels. Scant adipose tissue was identified at the periphery of the tumour, suggesting the possibility of AML. On immunohistochemistry (Fig. 4), the large polygonal cells were immunopositive for cytokeratin, epithelial membrane antigen, CD10 and vimentin, confirming them as RCC. The spindled and epithelioid cells were positive for $\mathrm{HMB}-45$ and smooth muscle actin, confirming the AML component. Tumour cell nuclei showed loss of hamartin expression (Fig. 4, part G), while tuberin expression was retained. The RCC component invaded the capsule and infiltrated the perinephric fat. Foci of necrosis, hyalinization and calcification were identified. Lymphovascular emboli from the RCC were present in the AML areas. Based on the histomorphogical and immuno- 


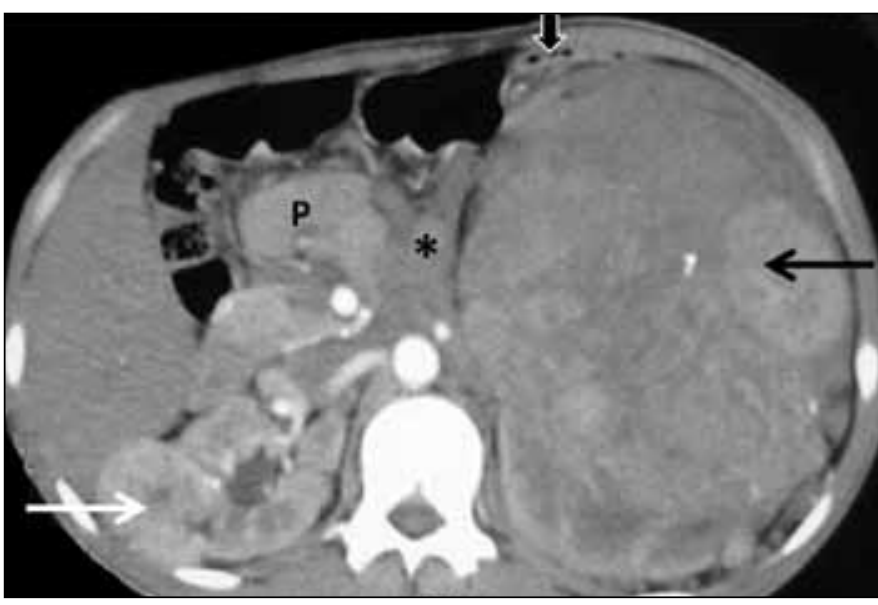

Fig. 1. Contrast enhanced CT axial section shows a large, well-defined, heterogeneously enhancing mass replacing the entire left kidney (black arrow) with intralesional areas of necrosis. The mass is displacing the small bowel loops $\left({ }^{*}\right)$ and pancreas (P) medially and the colon (block arrow) anteriorly. Another enhancing mass is noted arising from midpole of right kidney (white arrow). Both masses do not show any areas of calcification or fat components within.

histochemical features, a diagnosis of composite tumour, with clear cell RCC was made. As histopathology revealed an RCC component, he was followed-up according to the RCC follow-up protocol. At the 1-year follow-up, no recurrence was observed and the patient is currently doing well.

\section{Discussion}

TS is an autosomal dominant disorder characterized by mental retardation, epilepsy, and tumour-like hamartomas involving various organs. Renal pathology is encountered in about $60 \%$ of TS patients, including renal cysts, AMLs, and epithelial neoplasms viz, RCCs, and oncocytomas. The incidence of $\mathrm{AML}$ in TS is $80 \%$, whereas that of RCC in TS is $1 \%$ to $-2 \%{ }^{3,4}$ However, concomitant occurrence of RCC and $\mathrm{AML}$ is rare and there are about 60 reported cases. ${ }^{2}$ In TS patients, AML occurs at a younger age and tends to be larger, multicentric and bilateral. ${ }^{5}$ But this difference in age does not hold true in concurrent tumours in patients with and without TS. ${ }^{1}$ The mean age of TS patients with coexisting RCC and $\mathrm{AML}$ is 59 years, and a striking female predominance has been observed in comparison to sporadic cases. ${ }^{1}$ The present case is interesting as it occurred in a young male.

Most cases with concomitant tumours had discrete RCC and $\mathrm{AML}$ areas within the same kidney (collision tumour) or had synchronous occurrence of RCC in one kidney and AML in the other kidney. However, this distinction is not clear in all reports, and it is difficult to comment definitively if these tumours were collision, composite or bilateral synchronous ones. Only 15 cases have been reported, in which both the components were within the same tumour mass. ${ }^{2,6,7}$ Two of these had a sharply circumscribed lesion of RCC within an $\mathrm{AML},{ }^{2,3} 2$ were RCCs with AML at the periphery, with admixture of tumor components, ${ }^{6}$ and the remaining did not mention whether the RCC and AML components were intermingled..$^{7-9}$ In our case, both these components were closely intermingled (composite tumour), which adds to its rarity. Clear cell RCC has been described as the most common type of RCC both in sporadic as well as TS-associated cases of concurrent tumours, ${ }^{1}$ as is our presented case.

The significance of identifying concomitant RCC and AML is multi-pronged. On imaging, CT can usually distinguish between AML and RCC. AML usually includes a macroscopic lipid component, while RCC will appear as a heterogeneously enhancing mass. At times, an overlap on imaging can occur, as 5\% of AML are lipopenic and $10 \%$ of RCC can have lipid components. When the AML
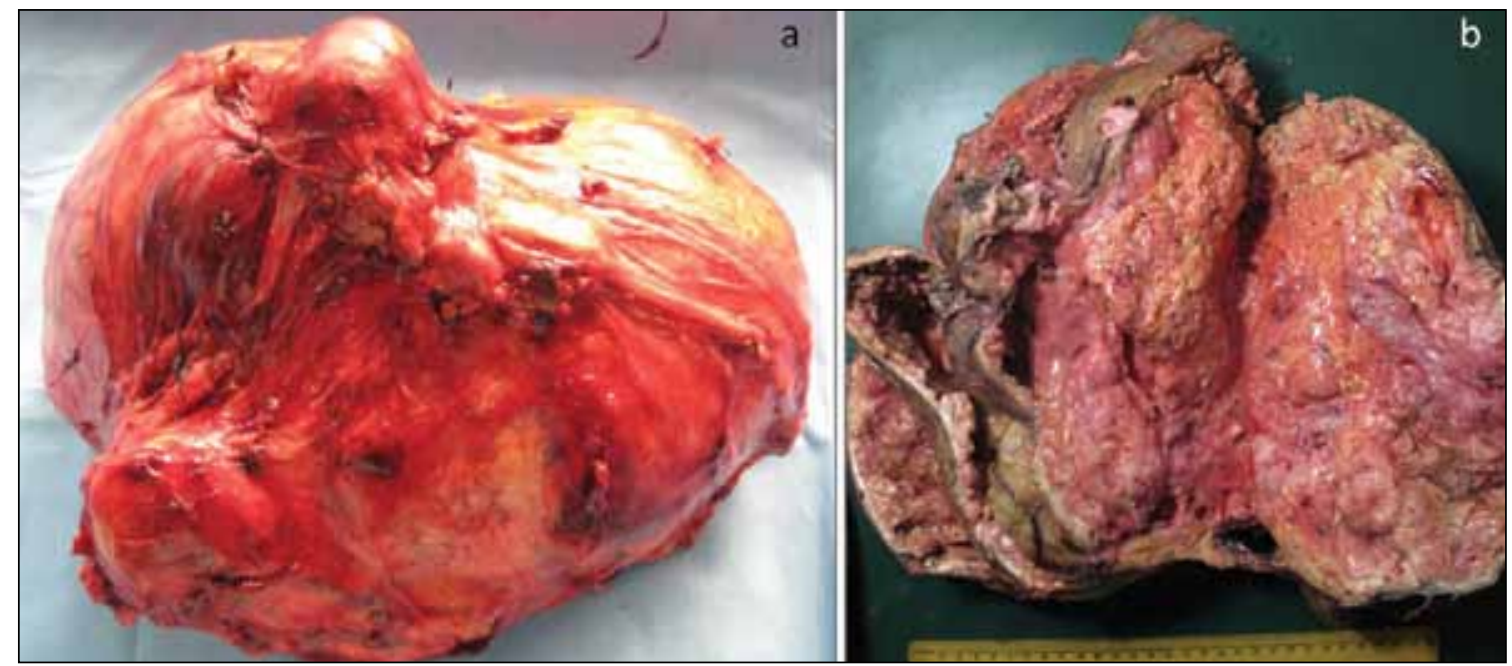

Fig. 2. Left nephrectomy specimen showing irregular enlargement of the kidney (a). Cut surface shows a large grey-white tumor, with areas of necrosis (b) 


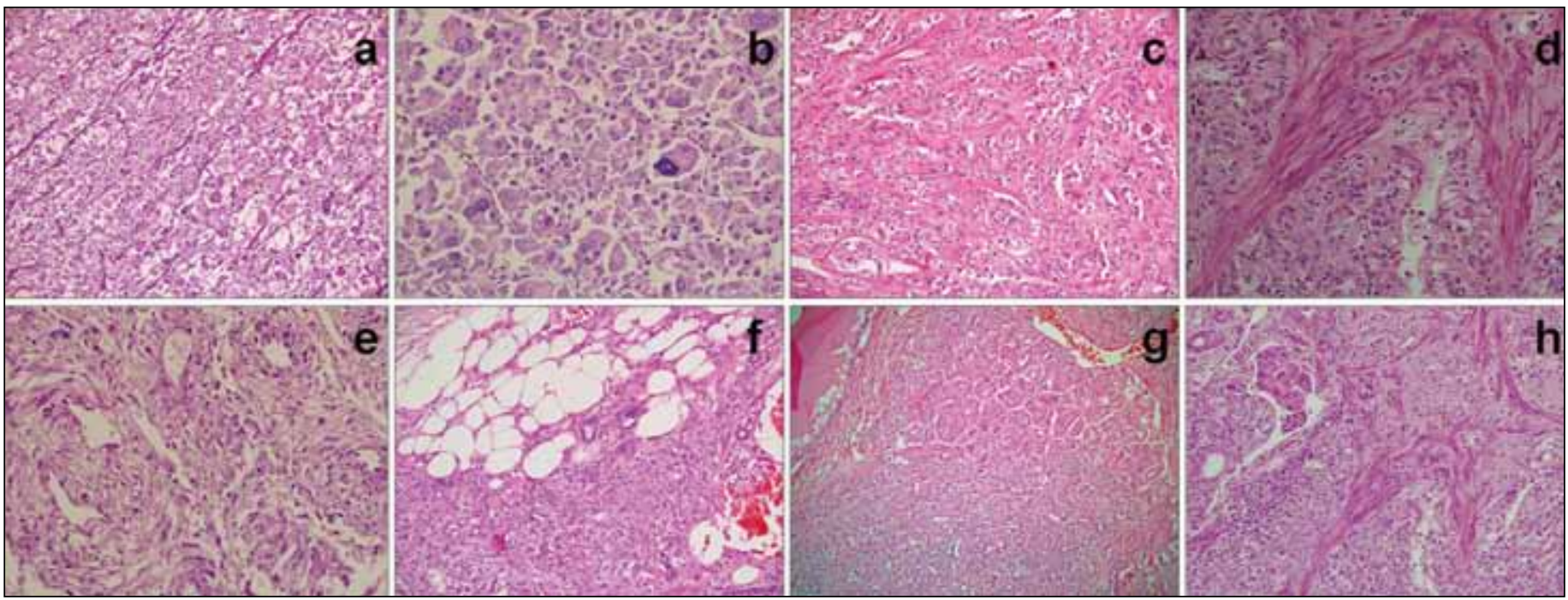

Fig. 3. Photomicrographs of HE stained sections show a renal cell carcinoma (RCC) with tumor cells arranged in nests separated by thin vascular septae (a; $x 200)$; high magnification shows marked nuclear pleomorphism ( $b ; x 400)$; areas of angiomyolipoma (AML) composed of spindled and small epithelioid cells (c; x200) with uniform round to oval nuclei are present $(d ; x 400)$; at places the spindled cells originate from walls of abnormal blood vessels $(e ; x 200)$. Foci of adipose tissue (f; $\mathrm{x} 200)$, intimate admixture of RCC and AML ( $;$; 100$)$, and lymphovascular tumor emboli $(\mathrm{h} ; \mathrm{x200})$ are observed.

component lacks significant lipomatous areas, radiological features may not be typical, leading to misinterpretation as RCC alone, as occurred in our case. Therefore, in such cases, MRI may be performed for further characterization, or tissue diagnosis should be obtained. On histopathological examination of a resected tumour specimen, the RCC component may be very small and is liable to be missed if the specimen is not adequately sampled. Thus, particularly in patients with TS, AMLs should be extensively sampled. The clinical outcome of coexisting AML and RCC depends on the pathologic stage of the RCC component. As rightly pointed out by Aron and colleagues, the lipomatous areas of the AML component may be mistaken for perinephric fat or renal sinus fat invasion in a classical RCC, resulting in upstaging of the tumour. ${ }^{6}$ A spindle cell predominant $A M L$, particularly one showing degenerative nuclear atypia, may be mistaken for sarcomatoid RCC, which again has worse outcomes. Further, epithelioid component of AML may be misinterpreted as RCC. Hence, immunohistochemistry for identification of AML is warranted if any unusual morphology is noted in an otherwise typical RCC, especially in TS patients. On core needle biopsy, only the AML component
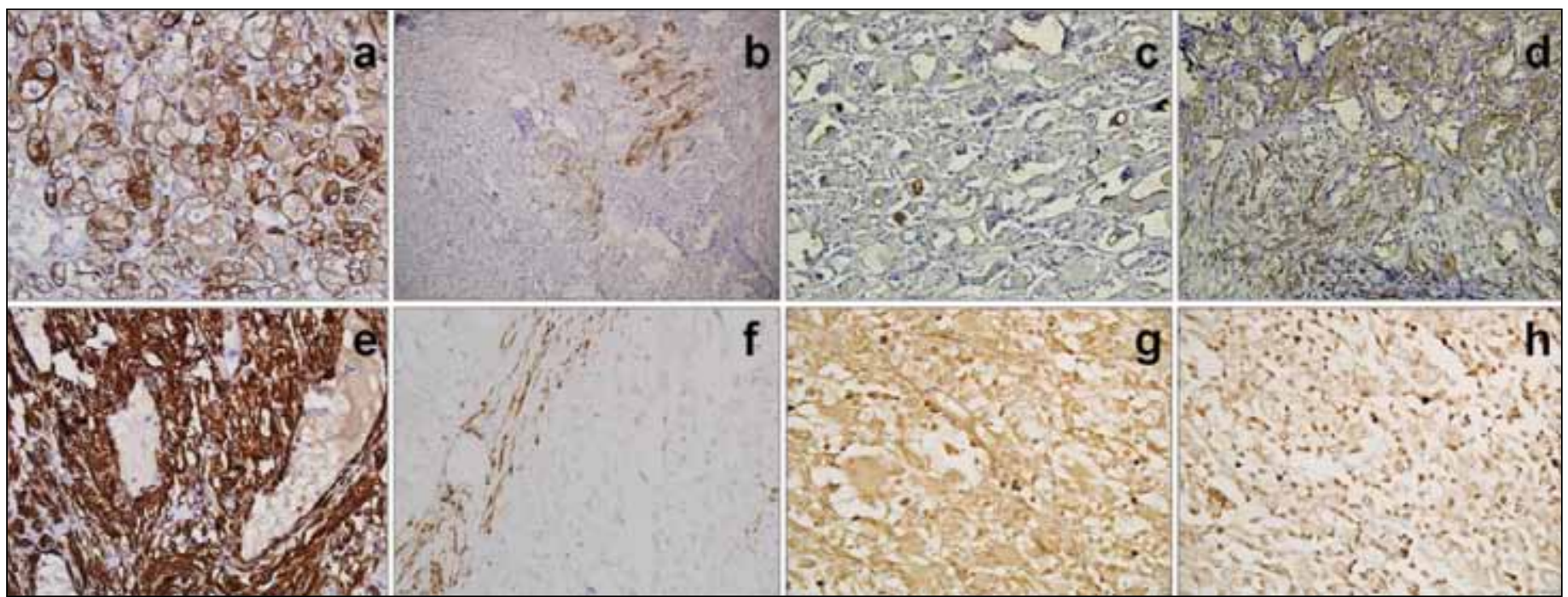

Fig. 4. Immunohistochemistry for CK is positive in the RCC areas $(a ; x 400)$ while negative in AML areas $(b ; x 200)$; focal EMA positivity is seen (c; $x 200)$; vimentin is positive in both RCC (above) and AML (below) components (d; $x 200)$; SMA $(e ; x 400)$ and HMB45 $(f ; x 400)$ are positive in AML component. Tumor cells show loss of nuclear expression of hamartin $(g ; x 400)$, while tuberin expression is retained $(h ; x 400)$. 
might be sampled without sampling admixed RCC, leading to a diagnosis of AML alone, thus, ultimately resulting in patient mismanagement.

AML is a benign condition. If the size is smaller than $4 \mathrm{~cm}$, the patient is kept under observation. However, in AML associated with TS, the possibility of a composite tumour should be considered. Fat component on CT is diagnostic of AML; however, small foci of RCC may be missed, as in our case. MRI is of help in equivocal cases, as it can depict microscopic fat in lipopenic AML, and can potentially differentiate between AML and RCC on the basis of apparent diffusion coefficient values in diffusion weighted imaging. Thus, a more vigorous surveillance protocol with frequent MRIs should be adopted for this group of patients if observation is planned.

\section{Conclusion}

Though the coexistence of RCC with AML is rare, and the composite variant is even rarer, one must be aware of this entity to prevent misdiagnosis as well as upstaging of RCC, and also to avoid missing areas of RCC within a large AML, especially in TS patients.

Competing interests: The authors all declare no competing financial or personal interests.

\section{References}

1. Jimenez RE, Eble JN, Reuter VE, et al. Concurrent angiomyolipoma and renal cell neoplasia: A study of 36 cases. Mod Pathol 2001;14:157-63. http://dx.doi.org/10.4103/1319-2442.109591

2. Hussain $M$, Mubarak $M$, Sultan $G$, et al. Renal transplant in a tuberous sclerosis patient with bilateral giant renal angiomyolipomas and concurrent renal carcinoma. Saudi I Kidney Dis Transpl 2013;24:318-21. http://dx.doi.org/10.4103/1319-2442.109591

3. Corsenca A, Aebersold F, Moch H, et al. Combined nephrectomy and pre-emptive renal transplantation in a tuberous sclerosis patient with angiomyolipoma, renal carcinoma and life-threatening abdominal hemorrhages. Nephrol Dial Transplant 2007;22:3330-33. http://dx.doi.org/10.1093/ndt/gfm552

4. Rakowski SK, Winterkorn EB, Paul E, et al. Renal manifestations of tuberous sclerosis complex: Incidence, prognosis, and predictive factors. Kidney Int 2006;70:1777-82. http://dx.doi.org/10.1038/ sj.ki.5001853

5. Nelson CP, Sanda MG. Contemporary diagnosis and management of renal angiomyolipoma: I Urol 2002;168:1315-25. http://dx.doi.org/10.1016/S0022-5347(05)64440-0

6. Aron $M$, Aydin $H$, Sercia $L$, et al. Renal cell carcinomas with intratumoral fat and concomitant angiomyolipoma: Potential piffalls in staging and diagnosis. Am J Clin Path 2010;134:807-12. http://dx.doi. org/10.1309/AJCP019JAPYRDTID

7. Tsuboniwa N, Meguro N, Nakamura Y, et al. Coexistence of renal cell carcinoma and renal angiomyolipoma developing in a kidney: A case report. Acta Urol Jpn 1997;43:131-35.

8. Mouded IM, Tolia BM, Bernie JE, et al.Symptomatic renal angiomyolipoma: Report of 8 cases, 2 with spontaneous rupture. J Urol 1978; 119:684-8.

9. Oesterling JE, Fishman EK, Goldman SM, et al. The management of renal angiomyolipoma. J Urol $1986 ; 135: 1121-24$.

Correspondence: Dr. Mehar C. Sharma, Professor, Department of Pathology, All India Institute of Medical Sciences, Ansari Nagar, New Delhi - 110029, India; sharmamehar@yahoo.co.in

This paper has been peer-reviewed. 